Research Paper

\title{
Platinum Agent-Induced Hypersensitivity Reactions: Data Mining of the Public Version of the FDA Adverse Event Reporting System, AERS
}

\author{
Toshiyuki Sakaeda ${ }^{1 凶}$, Kaori Kadoyama ${ }^{1}$, Hiroaki Yabuuchi ${ }^{2,3}$, Satoshi Niijima ${ }^{2}$, Kyoko Seki ${ }^{4}$, Yukinari \\ Shiraishi ${ }^{4}$ and Yasushi Okuno $2,3 \bowtie$ \\ 1. Center for Development of Integrative Education in Pharmacy and Pharmaceutical Sciences, Graduate School of Phar- \\ maceutical Sciences, Kyoto University, Kyoto 606-8501, Japan \\ 2. Department of Systems Biosciences for Drug Discovery, Graduate School of Pharmaceutical Sciences, Kyoto University, \\ Kyoto 606-8501, Japan \\ 3. Kyoto Constella Technologies Co., Ltd., Kyoto 604-8156, Japan \\ 4. Department of Pharmacy, Japan Labour Health and Welfare Organization, Kobe Rosai Hospital, Kobe 651-0053, Japan
}

Corresponding author: Toshiyuki Sakaeda, Ph.D., Center for Development of Integrative Education in Pharmacy and Pharmaceutical Sciences, Graduate School of Pharmaceutical Sciences, Kyoto University, Kyoto 606-8501, Japan, Tel: +81-75-753-9560, Fax: +81-75-753-4502, e-mail: sakaedat@pharm.kyoto-u.ac.jp; Yasushi Okuno, Ph.D., Department of Systems Biosciences for Drug Discovery, Graduate School of Pharmaceutical Sciences, Kyoto University, Kyoto 606-8501, Japan, Tel\&Fax: +81-75-753-4559, e-mail: okuno@pharm.kyoto-u.ac.jp

(C) Ivyspring International Publisher. This is an open-access article distributed under the terms of the Creative Commons License (http://creativecommons.org/ licenses/by-nc-nd/3.0/). Reproduction is permitted for personal, noncommercial use, provided that the article is in whole, unmodified, and properly cited.

Received: 2011.03.24; Accepted: 2011.05.20; Published: 2011.05.21

\begin{abstract}
Objective: Adverse event reports (AERs) submitted to the US Food and Drug Administration (FDA) were reviewed to confirm the platinum agent-associated mild, severe, and lethal hypersensitivity reactions.

Methods: Authorized pharmacovigilance tools were used for quantitative signal detection, including the proportional reporting ratio, the reporting odds ratio, the information component given by a Bayesian confidence propagation neural network, and the empirical Bayes geometric mean. Excess2, given by the multi-item gamma Poisson Shrinker algorithm, was used to evaluate the effects of dexamethasone and diphenhydramine on oxaliplatin-induced hypersensitivity reactions.

Results: Based on 1,644,220 AERs from 2004 to 2009, carboplatin and oxaliplatin proved to cause mild, severe, and lethal hypersensitivity reactions, whereas cisplatin did not. Dexamethasone affected oxaliplatin-induced mild hypersensitivity reactions, but had lesser effects on severe and lethal reactions. The effects of diphenhydramine were not confirmed.

Conclusion: The FDA's adverse event reporting system, AERS, with optimized data mining tools is useful to authorize potential associations between platinum agents and hypersensitivity reactions.
\end{abstract}

Key words: adverse event, AERS, platinum agent, hypersensitivity

\section{Introduction}

The treatment of metastatic colorectal cancer has progressed significantly over the past 20 years, and currently the FOLFIRI or FOLFOX regimen [1-4], with or without a targeted monoclonal antibody, is the standard treatment [5-8], consisting of the injection of a bolus of 5-fluorouracil (5-FU), irinotecan or oxali- 
platin (L-OHP), and the infusion of 5-FU/leucovorin, respectively. Future improvements will likely require the incorporation of or substitution with a novel anticancer drug, personalization based on genetic profiling, or pharmacokinetically-guided administration.

Hypersensitivity reactions are a well-known complication of the use of the platinum agents, cisplatin (CDDP) and carboplatin (CBDCA) [9-12]. $\mathrm{L}-\mathrm{OHP}$, a third-generation platinum agent, has also been increasingly recognized to cause hypersensitivity reactions [13-16], but the incidence still varies in reports [17-25]. It is difficult to evaluate the exact prevalence of these reactions, presumably because their definition is vast and pathogenic mechanisms are still vague, but L-OHP-induced hypersensitivity can be classified into relatively acute severe anaphylaxes and delayed mild allergic reactions [13-16]. A reduction of the infusion rate and the administration of steroids and/or antihistamines are used to treat both for acute and delayed hypersensitivity reactions, and discontinuation is strongly suggested immediately upon the development of acute reactions [13-16]. However, large-scale validation is still awaited.

In this study, adverse event reports (AERs) submitted to the US Food and Drug Administration (FDA) were reviewed to confirm the platinum agent-associated mild, severe, and lethal hypersensitivity reactions. This data base relies on spontaneous reports to the FDA generated by health professionals, consumers, and manufacturers, and the system is referred to as the Adverse Event Reporting System (AERS). The structure of AERS is in compliance with international safety reporting guidance, ICH E2B. Recently, the AERS database has been used for evaluation of safety profiles of statins [26-29], rofecoxib [30], topical bovine thrombin [31] and infliximab [32]. Here, the effects of dexamethasone and diphenhydramine on L-OHP-induced reactions were also evaluated to suggest a management strategy for patients with hypersensitivity reactions. The effects of bevacizumab, often used with L-OHP, were also evaluated.

\section{Methods}

The AERS database covers several million case reports on adverse events. Pharmacovigilance analysis aims to search for previously unknown patterns and automatically detect important signals, i.e., drug-associated adverse events, from such a large database. Recently developed data mining tools for pharmacovigilance have been successful at detecting signals that could not be found by individual case reviews and that warrant further investigation together with continuous surveillance. For this reason, data mining tools are being routinely used for pharmacovigilance, supporting signal detection and decision-making at companies, regulatory agencies, and pharmacovigilance centers [33-39]. Despite some limitations inherent to spontaneous reporting, the AERS database is a rich resource and the data mining tools described below provide a powerful means of identifying potential associations between drugs and adverse events.

\section{Data sources}

Input data for this study were taken from the public release of the FDA's AERS database, which covers the period from the first quarter of 2004 through the end of 2009. The database consists of 7 data sets; patient demographic and administrative information (DEMO), drug/biologic information (DRUG), adverse events (REAC), patient outcomes (OUTC), report sources (RPSR), drug therapy start and end dates (THER), and indications for use/diagnosis (INDI). The adverse events in REAC are coded using preferred terms (PTs) in the Medical Dictionary for Regulatory Activities (MedDRA) terminology.

Prior to analysis, all drug names were unified into generic names by a text-mining approach, because AERS permits the registering of arbitrary drug names, including trade names and abbreviations. For the batch conversion of drug names, reliable drug databases, e.g., FDA Orange Book, were utilized as a dictionary. Spelling errors were detected by GNU Aspell and carefully confirmed by working pharmacists. Furthermore, drug names which failed to receive generic names were manually converted to proper names. Foods, beverages, treatments (e.g. X-ray radiation), and unspecified names (e.g., beta-blockers) were omitted for this study. Duplicated reports were deleted according to FDA's recommendation of adopting the most recent CASE number (as described in one of the downloaded files, 'Asc_nts.doc' from the web-site of the FDA AERS database), resulting in the reduction of the number of AERs from 2,231,029 to $1,644,220$.

\section{Definition of adverse events}

According to the National Cancer Institute Common Terminology Criteria for Adverse Events (NCI-CTCAE) v4.0, AERs with PT10020751/ hypersensitivity in REAC were adopted as the reports on mild hypersensitivity reactions, in which 19 lower level terms (LLTs) were assigned in MedDRA v13.0, including LLT10000656/acute allergic reaction, LLT10001718/allergic reaction, LLT10020756/ hypersensitivity reaction, LLT10020759/ 
hypersensitivity symptom, LLT10038195/red neck syndrome, and LLT10046305/upper respiratory tract hypersensitivity reaction (site unspecified). AERs with PT10011906/death (with 13 LLT) or death terms in OUTC were excluded for mild hypersensitivity reactions. AERs with PT10002198/anaphylactic reaction were adopted as the reports on severe hypersensitivity reactions, in which 13 LLTs were assigned, including LLT10000663/acute anaphylactic reaction and LLT10002218/anaphylaxis. AERs both with PT10020751, and PT10011906 or death terms in OUTC were adopted as the reports on lethal hypersensitivity reactions. Of note, LLT10001718/allergic reaction and LLT10002218/anaphylaxis are assigned as allergic reactions and anaphylaxis in the NCI-CTCAE v4.0, respectively, and PTs in their higher levels were used in this study.

\section{Data mining}

In pharmacovigilance analysis, data mining algorithms have been developed to identify drug-associated adverse events as signals that are reported more frequently than expected by estimating expected reporting frequencies on the basis of information on all drugs and all events in the database [37-39]. For example, the proportional reporting ratio (PRR) [33], the reporting odds ratio (ROR) [34], the information component (IC) [35], and the empirical Bayes geometric mean (EBGM) [36] are widely used, and indeed, the PRR is currently used by the Medicines and Healthcare products Regulatory Agency (MHRA), UK, the ROR by the Netherlands Pharmacovigilance Centre, the IC by the World Health Organization (WHO), and the EBGM by the FDA.

All of these algorithms extract decision rules for signal detection and/or calculate scores to measure the associations between drugs and adverse events from a two-by-two frequency table of counts that involve the presence or absence of a particular drug and a particular event occurring in case reports. These algorithms, however, differ from one another in that the PRR and ROR are frequentist (non-Bayesian), whereas the IC and EBGM are Bayesian. In this section, only the scoring thresholds used in the present study are given, and the reader is referred to review articles for details [37-39].

For the PRR, a given drug-adverse event pair was defined as a signal, if the event count $\geq 3$, and the PRR $\geq 2.0$ with an associated chi-square value $\geq 4.0$ [33], and for the ROR, if the lower bound of the $95 \%$ two-sided confidence interval (CI) of ROR exceeded 1
[34]. For the IC, IC025, a criterion indicating the lower bound of the $95 \%$ two-sided CI of the IC, was adopted, and a IC025 value exceeding 0 was defined as a signal [35]. Lastly, for the EBGM, EB05 $\geq 2.0$ was set as a threshold for signal detection, where EB05 is interpreted as a lower one-sided 95\% confidence limit of EBGM [36].

The AERS database is also a valuable resource for exploring drug-drug interactions. Here, we are interested in how the co-administration of dexamethasone, diphenhydramine, and bevacizumab would affect L-OHP-induced mild, severe, and lethal hypersensitivity reactions, although the database does not provide the information on the timing of co-administration. To analyze such interactions, case reports on L-OHP were classified according to whether they also involved one of the three drugs. Any association among the interactions was then assessed using Excess2, a statistical index of the multi-item gamma Poisson shrinker (MGPS) algorithm [36].

\section{Results}

AERs in which CDDP, CBDCA, or L-OHP was the principal offending agent are summarized in Tables 1-3, and numbered 44,321, 39,653, and 33,194 of $1,644,220$, respectively. Reports of mild, severe, and lethal hypersensitivity reactions numbered 43,288, 18,225 , and 2,397, respectively.

CBDCA was administered in 229 of 43,288 AERs of mild, 72 of 18,225 AERs of severe, and 12 of 2,397 AERs of lethal hypersensitivity reactions (Table 2). L-OHP was administered in 126, 60 and 10, respectively (Table 3). The signals were detected for CBDCA and L-OHP by either the PRR, ROR, IC or EBGM, but no signal was suggested for CDDP (Table 1). The sensitivity was higher for ROR or IC, whereas lower for EBGM.

The effects of the co-administration of dexamethasone on L-OHP-induced hypersensitivity reactions are summarized in Table 4 . The values obtained with Excess2 were 18.66, 1.19 and -0.44, respectively, indicating that dexamethasone was more effective against mild than severe or lethal reactions. The effects of diphenhydramine were also examined, but no signal was detected (data not shown). The data on the co-administration of bevacizumab is listed in Table 5 . Values of Excess2 were 0.28, 5.38 and -5.65, respectively, and suggesting an effect of bevacizumab on severe L-OHP-induced reactions. 
Table 1. Signal detection for CDDP-associated mild, severe and lethal hypersensitivity reactions

\begin{tabular}{llll}
\hline & Mild & Severe & Lethal \\
& 43,288 & 18,225 & 2,397 \\
\hline No.of AERs & 38 & 29 & 5 \\
& & & \\
PRR & 0.436 & 0.790 & 1.036 \\
(kai2) & $(27.256)$ & $(1.412)$ & $(0.022)$ \\
& & & \\
ROR & 0.435 & 0.790 & 1.036 \\
$(95 \%$ two-sided CI) & $(0.317,0.553)$ & $(0.549,1.031)$ & $(0.431,1.641)$ \\
& & & -0.081 \\
IC & -1.195 & -0.353 & $(-1.287,1.125)$ \\
$(95 \%$ two-sided CI) & $(-1.651,-0.739)$ & $(-0.875,0.169)$ & 0.907 \\
& & & $(0.455)$ \\
\hline
\end{tabular}

Total number of adverse event reports (AERs) accompanied with CDDP administration was 44,321. Reports of mild, severe and lethal hypersensitivity reactions numbered 43,288, 18,225 and 2,397, respectively. PRR: the proportional reporting ratio [33], ROR: the reporting odds ratio [34], IC: the information component [35], EBGM: the empirical Bayes geometric mean [36]. There was no signal for CDDP-associated mild, severe and lethal hypersensitivity reactions (see "Methods" for the criteria of detection).

Table 2. Signal detection for CBDCA-associated mild, severe and lethal hypersensitivity reactions

\begin{tabular}{|c|c|c|c|}
\hline & $\begin{array}{l}\text { Mild } \\
43,288\end{array}$ & $\begin{array}{l}\text { Severe } \\
18,225\end{array}$ & $\begin{array}{l}\text { Lethal } \\
2,397\end{array}$ \\
\hline No.of AERs & 229 & 72 & 12 \\
\hline $\begin{array}{l}\text { PRR } \\
\text { (kai2) }\end{array}$ & $\begin{array}{l}2.949 * \\
(291.792)\end{array}$ & $\begin{array}{l}2.196^{*} \\
(45.698)\end{array}$ & $\begin{array}{l}2.780 * \\
(11.975)\end{array}$ \\
\hline $\begin{array}{l}\text { ROR } \\
\text { (95\% two-sided CI) }\end{array}$ & $\begin{array}{l}2.959 * \\
(2.598,3.320)\end{array}$ & $\begin{array}{l}2.201 \text { * } \\
(1.746,2.656)\end{array}$ & $\begin{array}{l}2.789 * \\
(1.582,3.996)\end{array}$ \\
\hline $\begin{array}{l}\text { IC } \\
(95 \% \text { two-sided CI) }\end{array}$ & $\begin{array}{l}1.539 * \\
(1.352,1.726)\end{array}$ & $\begin{array}{l}1.100 * \\
(0.767,1.433)\end{array}$ & $\begin{array}{l}1.233 * \\
(0.432,2.034)\end{array}$ \\
\hline $\begin{array}{l}\text { EBGM } \\
\text { ( } 95 \% \text { one-sided CI) }\end{array}$ & $\begin{array}{l}2.880 * \\
(2.580)\end{array}$ & $\begin{array}{l}2.097 \\
(1.723)\end{array}$ & $\begin{array}{l}2.079 \\
(1.288)\end{array}$ \\
\hline
\end{tabular}

Total number of adverse event reports (AERs) accompanied with CBDCA administrations was 39,653. Reports of mild, severe and lethal hypersensitivity reactions numbered 43,288, 18,225 and 2,397, respectively. PRR: the proportional reporting ratio [33], ROR: the reporting odds ratio [34], IC: the information component [35], EBGM: the empirical Bayes geometric mean [36]. *: signal detected, see "Methods" for the criteria of detection.

Table 3. Signal detection for L-OHP-associated mild, severe and lethal hypersensitivity reactions

\begin{tabular}{|c|c|c|c|}
\hline & $\begin{array}{l}\text { Mild } \\
43,288\end{array}$ & $\begin{array}{l}\text { Severe } \\
18,225\end{array}$ & $\begin{array}{l}\text { Lethal } \\
2,397\end{array}$ \\
\hline No.of AERs & 126 & 60 & 10 \\
\hline $\begin{array}{l}\text { PRR } \\
\text { (kai2) }\end{array}$ & $\begin{array}{l}1.934 \\
(55.797)\end{array}$ & $\begin{array}{l}2.186^{*} \\
(37.412)\end{array}$ & $\begin{array}{l}2.768^{*} \\
(9.604)\end{array}$ \\
\hline $\begin{array}{l}\text { ROR } \\
\text { (95\% two-sided CI) }\end{array}$ & $\begin{array}{l}1.937 \text { * } \\
(1.626,2.248)\end{array}$ & $\begin{array}{l}2.190 \text { * } \\
(1.699,2.681)\end{array}$ & $\begin{array}{l}2.775 \text { * } \\
(1.491,4.059)\end{array}$ \\
\hline $\begin{array}{l}\text { IC } \\
(95 \% \text { two-sided CI) }\end{array}$ & $\begin{array}{l}0.933 \text { * } \\
(0.681,1.185)\end{array}$ & $\begin{array}{l}1.087 \text { * } \\
(0.723,1.451)\end{array}$ & $\begin{array}{l}1.187 \text { * } \\
(0.312,2.062)\end{array}$ \\
\hline $\begin{array}{l}\text { EBGM } \\
\text { (95\% one-sided CI) }\end{array}$ & $\begin{array}{l}1.888 \\
(1.628)\end{array}$ & $\begin{array}{l}2.070 \\
(1.669)\end{array}$ & $\begin{array}{l}1.983 \\
(1.178)\end{array}$ \\
\hline
\end{tabular}

Total number of adverse event reports (AERs) accompanied with L-OHP administrations was 33,194. Reports of mild, severe and lethal hypersensitivity reactions numbered $43,288,18,225$ and 2,397, respectively. PRR: the proportional reporting ratio [33], ROR: the reporting odds ratio [34], IC: the information component [35], EBGM: the empirical Bayes geometric mean [36]. *: signal detected, see "Methods" for the criteria of detection. 
Table 4. Effect of co-administration of dexamethasone on L-OHP-associated hypersensitivity reactions

\begin{tabular}{|c|c|c|c|c|c|}
\hline & \multirow[t]{2}{*}{ L-OHP } & \multirow[t]{2}{*}{ dexamethasone } & \multicolumn{2}{|c|}{ Hypersensitivity } & \multirow[t]{2}{*}{ Excess2 } \\
\hline & & & yes & no & \\
\hline \multirow[t]{4}{*}{ Mild } & yes & yes & 40 & 4,774 & 18.66 \\
\hline & yes & no & 469 & 172,768 & \\
\hline & no & yes & 1,884 & 924,995 & \\
\hline & no & no & 132,784 & $184,631,220$ & \\
\hline \multirow[t]{4}{*}{ Severe } & yes & yes & 13 & 4,801 & 1.19 \\
\hline & yes & no & 214 & 173,023 & \\
\hline & no & yes & 919 & 925,960 & \\
\hline & no & no & 53,827 & $184,710,177$ & \\
\hline \multirow[t]{4}{*}{ Lethal } & yes & yes & 6 & 4,808 & -0.44 \\
\hline & yes & no & 54 & 173,183 & \\
\hline & no & yes & 393 & 926,486 & \\
\hline & no & no & 13,287 & $184,750,717$ & \\
\hline
\end{tabular}

The numbers of L-OHP-associated hypersensitivity reactions are listed. The interaction was assessed using Excess2, a statistical index of the multi-item gamma Poisson shrinker (MGPS) algorithm [36]. The data suggested that dexamethasone affected mild L-OHP-induced hypersensitivity reactions, but had lesser effects on severe and lethal reactions.

Table 5. Effect of co-administration of bevacizumab on L-OHP-associated hypersensitivity reactions

\begin{tabular}{|c|c|c|c|c|c|}
\hline & \multirow[t]{2}{*}{ L-OHP } & \multirow[t]{2}{*}{ bevacizumab } & \multicolumn{2}{|c|}{ Hypersensitivity } & \multirow[t]{2}{*}{ Excess2 } \\
\hline & & & yes & no & \\
\hline \multirow[t]{4}{*}{ Mild } & yes & yes & 35 & 11,943 & 0.28 \\
\hline & yes & no & 286 & 200,959 & \\
\hline & no & yes & 474 & 165,599 & \\
\hline & no & no & 134,382 & $185,355,256$ & \\
\hline \multirow[t]{4}{*}{ Severe } & yes & yes & 20 & 11,958 & 5.38 \\
\hline & yes & no & 103 & 201,142 & \\
\hline & no & yes & 207 & 165,866 & \\
\hline & no & no & 54,643 & $185,434,995$ & \\
\hline \multirow[t]{4}{*}{ Lethal } & yes & yes & 4 & 11,974 & -5.65 \\
\hline & yes & no & 45 & 201,200 & \\
\hline & no & yes & 56 & 166,017 & \\
\hline & no & no & 13,635 & $185,476,003$ & \\
\hline
\end{tabular}

The numbers of L-OHP-associated hypersensitivity reactions are listed. The interaction was assessed using Excess2, a statistical index of the multi-item gamma Poisson shrinker (MGPS) algorithm [36]. The data suggested that bevacizumab possibly affected severe L-OHP-induced hypersensitivity reactions.

\section{Discussion}

Although the exact mechanism by which platinum agents cause hypersensitivity reactions remains unclear, the agents are thought to induce a type I response mediated by IgE, followed by the release of histamine and cytokines, since reactions usually occur after multiple infusions [13-16]. Recent studies have suggested the involvement of a type IV reaction, i.e., T-cell-mediated production of cytokines, such as tumor necrosis factor-alpha and interleukin-6, especially for CDDP and CBDCA [13-16]. As far as L-OHP is concerned, most reactions are thought to be of type I, but reports of hemolysis and thrombocytopenia suggest a type II reaction, and chronic urticaria, joint pain and proteinuria can be attributed to a type III reaction [13-16]. The incidence of hypersensitivity reactions varies in reports, and this study was conducted to confirm the platinum agent-associated mild, severe, and lethal hypersensitivity reactions. Here, using an extremely large number of AERs submitted to the FDA with authorized data mining tools, CBDCA and 
L-OHP proved to cause mild, severe, and lethal hypersensitivity reactions, whereas CDDP did not.

Spontaneous reports of suspected adverse events are a valuable tool. However, this database has its limitations [37]. First, the data occasionally contain misspelling and miswords, although the structure of AERS is in compliance with the international safety reporting guidance. Second, the system was started more than 10 years ago, and reporting patterns have changed over time. Third, the adverse events are coded using hierarchical terms of PTs of MedDRA, and changes in terminology over time also might affect the quality of the database. Last, there are a number of duplicate entries in the database. To overcome problems with data quality, we manually corrected mistakes in the data entities and deleted duplicates according to FDA's recommended method. A long-term discussion on pharmacovigilance strategies with large numbers of spontaneous reports resulted in the quantitative signal detection indices PRR, ROR, IC and EBGM. Comparisons in terms of specificity showed that none of these indices is universally better than the others $[34,37,38]$, but EBGM is of lowest sensitivity in this study (Tables 2, 3).

Since 1998, the FDA has been exploring the MGPS program, which evaluates the signals for pairs and higher-order [35]. This program is used to detect possible synergistic interactions between drugs, i.e., drug-drug interaction. With an index of Excess2, the effects of dexamethasone and diphenhydramine on L-OHP-induced hypersensitivity reactions were evaluated to suggest the best patient management strategy. It was suggested that the co-administration of dexamethasone affected mild L-OHP-induced reactions more effectively, than severe or lethal reactions (Table 4). Here, the effects of diphenhydramine were not confirmed, but unexpectedly, it was suggested that bevacizumab affected L-OHP-induced severe reactions. It is noted that the database does not provide the information on the timing of co-administration. Additionally, we do not have the criteria, e.g., threshold value, of Excess2 to detect an unknown drug-drug interaction, and the calibration using many known drug-drug interactions would be necessary.

In conclusion, AERs submitted to the FDA were reviewed to confirm the platinum agent-associated mild, severe, and lethal hypersensitivity reactions. Authorized pharmacovigilance tools were used for quantitative signal detection, and the effects of dexamethasone and diphenhydramine on L-OHP-induced hypersensitivity reactions were also evaluated. Based on 1,644,220 AERs from 2004 to 2009, CBDCA and L-OHP proved to cause mild, severe, and lethal hy- persensitivity reactions, whereas CDDP did not. Dexamethasone affected L-OHP-induced mild hypersensitivity reactions, but had lesser effects on severe and lethal reactions.

\section{Conflict of Interest}

The authors have declared that no conflict of interest exists.

\section{References}

1. Douillard JY, Cunningham D, Roth AD, et al. Irinotecan combined with fluorouracil compared with fluorouracil alone as first-line treatment for metastatic colorectal cancer: a multicentre randomised trial. Lancet. 2000; 355: 1041-1047.

2. Tournigand C, André T, Achille E, et al. FOLFIRI followed by FOLFOX6 or the reverse sequence in advanced colorectal cancer: A randomized GERCOR study. J Clin Oncol. 2004; 22: 229-237.

3. de Gramont A, Figer A, Seymour M, et al. Leucovorin and fluorouracil with or without oxaliplatin as first-line treatment in advanced colorectal cancer. J Clin Oncol. 2000; 18: 2938-2947.

4. Goldberg RM, Sargent DJ, Morton RF, et al. A randomized controlled trial of fluorouracil plus leucovorin, irinotecan, and oxaliplatin combinations in patients with previously untreated metastatic colorectal cancer. J Clin Oncol. 2004; 22: 23-30.

5. Grothey A, Sargent D, Goldberg RM, et al. Survival of patients with advanced colorectal cancer improves with the availability of fluorouracil-leucovorin, irinotecan, and oxaliplatin in the course of treatment. J Clin Oncol. 2004; 22: 1209-1214.

6. Venook A. Critical evaluation of current treatments in metastatic colorectal cancer. Oncologist. 2005; 10: 250-261.

7. Lee JJ, Chu E. An update on treatment advances for the first-line therapy of metastatic colorectal cancer. Cancer J. 2007; 13: 276-281.

8. Sabharwal A, Kerr D. Chemotherapy for colorectal cancer in the metastatic and adjuvant setting: past, present and future. Expert Rev Anticancer Ther. 2007; 7: 477-487.

9. Shepherd GM. Hypersensitivity reactions to chemotherapeutic drugs. Clin Rev Allergy Immunol. 2003; 24: 253-262.

10. Heywood GR, Rosenberg SA, Weber JS, et al. Hypersensitivity reactions to chemotherapy agents in patients receiving chemoimmunotherapy with high-dose interleukin 2. J Natl Cancer Inst. 1995; 87: 915-922.

11. Markman M, Kennedy A, Webster K, et al. Clinical features of hypersensitivity reactions to carboplatin. J Clin Oncol. 1999; 17: 1141-1145.

12. Markman M. Toxicities of the platinum antineoplastic agents. Expert Opin Drug Saf. 2003; 2: 597-607.

13. Syrigou E, Syrigos K, Saif MW. Hypersensitivity reactions to oxaliplatin and other antineoplastic agents. Curr Allergy Asthma Rep. 2008; 8: 56-62

14. Eng C. Toxic effects and their management: daily clinical challenges in the treatment of colorectal cancer. Nat Rev Clin Oncol. 2009; 6: 207-218.

15. Makrilia N, Syrigou E, Kaklamanos I, et al. Hypersensitivity reactions associated with platinum antineoplastic agents: a systematic review. Met Based Drugs 2010; 2010: 207084.

16. Lee C, Gianos M, Klaustermeyer WB. Diagnosis and management of hypersensitivity reactions related to common cancer chemotherapy agents. Ann Allergy Asthma Immunol. 2009; 102: 179-187.

17. Andre T, Boni C, Mounedji-Boudiaf L, et al. Multicenter international study of oxaliplatin/5-fluorouracil/leucovorin in the adjuvant treatment of colon cancer (MOSAIC) investigators, 
oxaliplatin, fluorouracil, and leucovorin as adjuvant treatment for colon cancer. N Engl J Med. 2004; 350: 2343-2351.

18. Thomas RR, Quinn MG, Schuler B, et al. Hypersensitivity and idiosyncratic reactions to oxaliplatin. Cancer 2003; 97: 2301-2307.

19. Siu SW, Chan RT, Au GK, et al. Hypersensitivity reactions to oxaliplatin: experience in a single institute. Ann Oncol. 2006; 17: 259-261.

20. Maindrault-Goebel F, Andre T, Tournigand C, et al. Allergic-type reactions to oxaliplatin: retrospective analysis of 42 patients. Eur J Cancer 2005; 41: 2262-2267.

21. Gowda A, Goel R, Berdzik J, et al. Hypersensitivity reactions to oxaliplatin: incidence and management. Oncology. 2004; 18: 1671-1675.

22. Brandi G, Pantaleo MA, Galli C, et al. Hypersensitivity reactions related to oxaliplatin (OHP). Br J Cancer. 2003; 89: 477-481.

23. Shibata Y, Ariyama H, Baba E, et al. Oxaliplatin-induced allergic reaction in patients with colorectal cancer in Japan. Int J Clin Oncol. 2009; 14: 397-401.

24. Seki K, Senzaki K, Tsuduki Y, et al. Multicenter trial on hypersensitivity reactions following treatment with FOLFOX regimens. Jpn J Pharm Health Care Sci. 2008; 34: 919-926.

25. Seki K, Senzaki K, Tsuduki Y, et al. Risk factors for oxaliplatin-induced hypersensitivity reactions in Japanese patients with advanced colorectal cancer. Int J Med Sci. 2010; 8: 210-215.

26. Alsheikh-Ali AA, Ambrose MS, Kuvin JT, et al. The safety of rosuvastatin as used in common clinical practice: a postmarketing analysis. Circulation. 2005; 111: 3051-3057.

27. Zipes DP, Zvaifler NJ, Glassock RJ, et al. Rosuvastatin: an independent analysis of risks and benefits. MedGenMed. 2006; 8: 73.

28. Holoshitz N, Alsheikh-Ali AA, Karas RH. Relative safety of gemfibrozil and fenofibrate in the absence of concomitant cerivastatin use. Am J Cardiol. 2008; 101: 95-97.

29. Colman E, Szarfman A, Wyeth J, et al. An evaluation of a data mining signal for amyotrophic lateral sclerosis and statins detected in FDA's spontaneous adverse event reporting system. Pharmacoepidemiol Drug Saf. 2008; 17: 1068-1076.

30. Bottone FGJr, Barry WT. Postmarketing surveillance of serious adverse events associated with the use of rofecoxib from 1999-2002. Curr Med Res Opin. 2009; 25: 1535-1550.

31. Clark JA, Humphries JE, Crean S, et al. Topical bovine thrombin: a 21-year review of topical bovine thrombin spontaneous case safety reports submitted to FDA's Adverse Event Reporting System. Pharmacoepidemiol Drug Saf. 2010; 19: 107-114.

32. Hansen RA, Gartlehner G, Powell GE, et al. Serious adverse events with infliximab: analysis of spontaneously reported adverse events. Clin Gastroenterol Hepatol. 2007; 5: 729-735.

33. Evans SJ, Waller PC, Davis S. Use of proportional reporting ratios (PRRs) for signal generation from spontaneous adverse drug reaction reports. Pharmacoepidemiol Drug Saf. 2001; 10: 483-486

34. van Puijenbroek EP, Bate A, Leufkens HG, et al. A comparison of measures of disproportionality for signal detection in spontaneous reporting systems for adverse drug reactions. Pharmacoepidemiol Drug Saf. 2002; 11: 3-10.

35. Bate A, Lindquist $M$, Edwards IR, et al. A Bayesian neural network method for adverse drug reaction signal generation. Eur J Clin Pharmacol. 1998; 54: 315-321.

36. Szarfman A, Machado SG, O'Neill RT. Use of screening algorithms and computer systems to efficiently signal higher-than-expected combinations of drugs and events in the US FDA's spontaneous reports database. Drug Saf. 2002; 25: 381-392.

37. Bate A, Evans SJ. Quantitative signal detection using spontaneous ADR reporting. Pharmacoepidemiol Drug Saf. 2009; 18 : 427-436.
38. Gould AL. Practical pharmacovigilance analysis strategies. Pharmacoepidemiol Drug Saf. 2003; 12: 559-574.

39. Almenoff JS, Pattishall EN, Gibbs TG, et al. Novel statistical tools for monitoring the safety of marketed drugs. Clin Pharmacol Ther. 2007; 82:157-166. 